INPLASY

PROTOCOL

To cite: Deng et al.

Effectiveness of moxibustion therapy in the Treatment of

Urticaria: a protocol for a systematic review and metaanalysis. Inplasy protocol 2020100040. doi:

10.37766/inplasy2020.10.0040

Received: 12 October 2020

Published: 12 October 2020

Corresponding author:

Gen Deng

1084053154@qq.com

Author Affiliation:

Jiangxi University of

Traditional Chinese Medicine

Support: Jiangxi Education

Department.

Review Stage at time of this submission: Preliminary searches.

Conflicts of interest:

No conflict of interest.

\section{Effectiveness of moxibustion therapy in the Treatment of Urticaria: a protocol for a systematic review and meta-analysis}

Deng, G1; Ye, W2; Wan, Q3; Wang, J4.

Review question / Objective: How about the efficacy and safety of moxibustion in the treatment of urticaria.

Condition being studied: Urticaria is a common skin disease in clinic. The main clinical symptoms are sudden attack, various forms, different sizes of wind and erythema, accompanied by varying degrees of itching. At present, antihistamines, non-specific antiallergic agents or glucocorticoids are the main treatment, with some side effects and adverse reactions. Moxibustion therapy has shown strong advantages in the treatment of urticaria, and the curative effect is accurate. therefore, this paper will carry out a systematic evaluation and meta analysis of the efficacy and safety of moxibustion in the treatment of urticaria.

INPLASY registration number: This protocol was registered with the International Platform of Registered Systematic Review and Meta-Analysis Protocols (INPLASY) on 12 October 2020 and was last updated on 12 October 2020 (registration number INPLASY2020100040).

\section{INTRODUCTION}

Review question / Objective: How about the efficacy and safety of moxibustion in the treatment of urticaria.

Rationale: A systematic evaluation and meta analysis of clinical randomized controlled studies on moxibustion for urticaria following the rules of evidencebased medicine.

Condition being studied: Urticaria is a common skin disease in clinic. The main clinical symptoms are sudden attack, various forms, different sizes of wind and erythema, accompanied by varying degrees of itching. At present, antihistamines, non- 
specific antiallergic agents or glucocorticoids are the main treatment, with some side effects and adverse reactions. Moxibustion therapy has shown strong advantages in the treatment of urticaria, and the curative effect is accurate. therefore, this paper will carry out a systematic evaluation and meta analysis of the efficacy and safety of moxibustion in the treatment of urticaria.

\section{METHODS}

Search strategy: Eight electronic databases will be searched, including PubMed, Excerpta Medica Database, Web of Science, Cochrane Library, the China National Knowledge Infrastructure(CNKI), Chinese Science and Technology Periodical Database(VIP), Wanfang Database(WF), and Chinese Biomedical Literature Database(CBM). We will search above electronic databases from the beginning to October 2020, without any language restriction, but involving only the human subjects.

Participant or population: There are clear and recognized diagnostic criteria and efficacy criteria, and all patients are diagnosed as urticaria, regardless of gender, age and origin of the case.

Intervention: Moxibustion therapy will include all therapies using any type of moxibustion, such as indirect moxibustion, direct moxibustion, heat-sensitive moxibustion, and so on. Mixed therapies based on moxibustion will also be included.

Comparator: The control group will receive one of the following treatment methods: conventional pharma-cological therapy, no treatment, and placebo.

Study designs to be included: Clinical randomized controlled trials (RCTs) containing moxibustion for urticaria were included.

Eligibility criteria: 1. Inclusion criteria for study selection 1.1. Types of studies. Clinical randomized controlled trials (RCTs) containing moxibustion for urticaria were included, with no limitation of language and publication status. 1.2. Types of participants. There are clear and recognized diagnostic criteria and efficacy criteria, and all patients are diagnosed as urticaria, regardless of gender, age and origin of the case. 1.3. Types of interventions 1.3.1. Experimental interventions. Moxibustion therapy will include all therapies using any type of moxibustion, such as indirect moxibustion, direct moxibustion, heat-sensitive moxibustion, and so on. Mixed therapies based on moxibustion will also be included. 1.3.2. Control interventions. The control group will receive one of the following treatment methods: conventional pharma-cological therapy, no treatment, and placebo. RCTs comparing different types of moxibustion therapy will be excluded. 1.4. Types of outcome measures. 1.4.1. Primary outcome. Clinical efficacy, including total effective rate or cure rate, and recurrence rate will be accepted as the primary outcomes. 1.4.2. secondary outcomes. The itch level, number of clusters, size of clusters, and laboratory test results will be used as secondary outcomes.

Information sources: Eight electronic databases will be searched, including PubMed, Excerpta Medica Database, Web of Science, Cochrane Library, the China National Knowledge Infrastructure(CNKI), Chinese Science and Technology Periodical Database(VIP), Wanfang Database(WF), and Chinese Biomedical Literature Database(CBM). We will search above electronic databases from the beginning to October 2020, without any language restriction, but involving only the human subjects.

Main outcome(s): Clinical efficacy, including total effective rate or cure rate, and recurrence rate will be accepted as the primary outcomes.

Additional outcome(s): The itch level, number of clusters, size of clusters, and laboratory test results will be used as secondary outcomes. 
Data management: The two researchers independently read the title and abstract of the literature they obtained, read the full text of the trials that might meet the inclusion criteria to determine whether the inclusion criteria were truly met, and discussed the conflicting literatures or let the third researcher decide whether to include them. Two researchers independently extracted data from the included studies, including study design, intervention measures and methods, measurement indicators, results, methodological contents such as hidden grouping and blind method, etc., and a third evaluator checked the consistency of the data. If the required information is incomplete, we will contact the original author for the required data.

Quality assessment / Risk of bias analysis: The Cochrane Handbook of Systematic Review (5.1.0) RCT risk assessment tool was used to evaluate the risk of bias by two independent researchers, including: (1) The method of random sequence generation; (2) Allocation hiding; (3) Whether the subjects and the implementer of the treatment plan should be blinded; (4) Blind method shall be applied to evaluators; (5) Integrity of result data; (6) Selective reporting of results; (7) Other bias. According to the results of each study that meets the inclusion criteria, according to the above 7 items, objectively judge that each study is high-risk or low-risk or unclear (no relevant information or uncertainty of bias is mentioned in the literature) and explain the reasons. If there are any differences in the above quality evaluation and data extraction process, two people shall discuss and resolve the differences or consult the third reviewer to deal with the differences.

Strategy of data synthesis: RevMan5.3 software will be used for statistical analysis. The odds ratio (OR) and its $95 \%$ Confidence Interval ( $\mathrm{Cl})$ will be used as the counting data, while the weighted mean difference (WMD) and its $95 \% \mathrm{Cl}$ will be used as the measurement data.
Subgroup analysis: If significant heterogeneity is found in our systematic review and sufficient data is available, we will conduct a subgroup analysis based on moxibustion type, moxibustion time, treatment cycle, and outcome measurement methods in the experimental and control groups.

Sensibility analysis: When sufficient RCTs are available, we will conduct sensitivity analysis by excluding low-quality or highquality studies one by one according to methodological quality, sample size and missing data.

Language: No limitation of language.

Country(ies) involved: China.

Keywords: Moxibustion ; Urticaria; Systematic Evaluation and meta Analysis.

Contributions of each author:

Author 1 - Gen Deng - The author drafted the manuscript.

Author 2 - Wenguo Ye - The author provided statistical expertise.

Author 3 - Qun Wan - The author contributed to the development of the selection criteria, and the risk of bias assessment strategy.

Author 4 - Jinlong Wang - The author read, provided feedback and approved the final manuscript. 